\title{
Protective effect of rosuvastatin treatment by regulating oxidized low-density lipoprotein expression in a rat model of liver fibrosis
}

\author{
SHUIPING YU ${ }^{1}$, XUELING ZHOU ${ }^{1}$, BINGZONG HOU ${ }^{2}$, BO TANG ${ }^{1},{\text { JIAN } \mathrm{LI}^{2} \text { and BAIMENG ZHANG }}^{2}$ \\ ${ }^{1}$ Department of Hepatobiliary Surgery, The Affiliated Hospital of Guilin Medical University, \\ Guilin, Guangxi 541000; ${ }^{2}$ Department of General Surgery, The Fifth Affiliated Hospital \\ of Sun Yat Sen University, Zhuhai, Guangdong 519000, P.R. China
}

Received February 12, 2016; Accepted April 27, 2016

DOI: $10.3892 /$ br.2016.722

\begin{abstract}
The present study aimed to evaluate the protective effect of rosuvastatin treatment on the mechanism of oxidized low-density lipoprotein (Ox-LDL) in rats with liver fibrosis. In total, 72 male Sprague-Dawley rats were divided into 3 groups: 24 in the control group (A), 24 in the obstructive jaundice models group (B) and 24 in the rosuvastatin group (C). Each group was further divided into four subgroups for assessment at different time-points. The obstructive jaundice models were established and rosuvastatin was administered by gavage. Liver fibrosis indicators, Ox-LDL, malonaldehyde (MDA) and superoxide dismutase (SOD), were measured and liver pathological changes were observed at weeks 1, 2, 3 and 4 after model induction. In groups B and $\mathrm{C}$, the rat models were successfully established, and there were significant changes in the expression of Ox-LDL and the three liver fibrosis indicators when compared to group A $(\mathrm{P}<0.01)$. However, the expression of $\mathrm{Ox}-\mathrm{LDL}$ and the three liver fibrosis indicators in group $\mathrm{C}$ were decreased compared with group $\mathrm{B}(\mathrm{P}<0.05)$, while SOD increased $(\mathrm{P}<0.05)$ and MDA decreased $(\mathrm{P}<0.05)$. The three liver fibrosis indicators were different in comparison to group $\mathrm{B}(\mathrm{P}<0.05)$. Thus, there appeared to be an association between the expression of Ox-LDL and liver fibrosis. Treatment with rosuvastatin could regulate the expression of Ox-LDL and improve liver fibrosis in rat models with obstructive jaundice.
\end{abstract}

\section{Introduction}

Liver fibrosis, a primary procedure of cirrhosis, is a progressive chronic procedure characterized by the accumulation of

Correspondence to: Dr Shuiping Yu, Department of Hepatobiliary Surgery, The Affiliated Hospital of Guilin Medical University, 15 Lequn Road, Guilin, Guangxi 541000, P.R. China

E-mail:478517575@qq.com

Key words: obstructive jaundice model, oxidized low-density lipoprotein, rosuvastatin, liver fibrosis extracellular matrix (ECM), which leads to serious damage to human health. Numerous studies have reported that fibrosis cannot be reversed; however, if the generation could be suppressed or degradation could be promoted of the ECM, liver fibrosis is reversible $(1,2)$. Therefore, it is important to explore the mechanism of delaying or improving liver fibrosis.

For the purpose of providing criterions for the treatment of liver fibrosis, certain studies are in process to discover the mechanism of liver fibrosis. Specifically, in obstructive jaundice rat models, the level of oxidized low-density lipoprotein (Ox-LDL) was previously considered to have a key role in atherosclerosis, the presence of the Ox-LDL-specific receptor in liver endothelial cells and had an important role in liver fibrosis $(3,4)$. Additionally, malonaldehyde (MDA) and superoxide dismutase (SOD) are signaling receptors that have specific roles in lipid peroxidation and functions as liver fibrosis regulators $(5,6)$. The level of Ox-LDL is known to increase in the liver and is correlated with the level of liver fibrosis. These evidences had a conclusion that Ox-LDL, MDA and SOD may be involved in improving liver fibrosis. Despite considerable evidence, the potential mechanism of liver fibrosis remains to be elucidated.

Early drug intervention can dominate the levels of Ox-LDL. For instance, rosuvastatin could reduce the levels of Ox-LDL in the plasma of hemodialysis patients with end-stage renal disease (7). Rosuvastatin could control the progress of liver fibrosis by inhibiting the activity of 3-hydroxy-3-methylglutaryl-coenzyme A (HMG-CoA), which is a key enzyme in the accumulation of cholesterol (8). It had been reported that rosuvastatin treatment could reduce the plasma concentrations of endogenous peroxidase and peroxidase activity, and regulate the expression of Ox-LDL in Wistar rats in 24 weeks (9). However, the underlying mechanism combined with rosuvastatin on liver fibrosis remains to be elucidated.

The present study aimed to show the association between rosuvastatin and liver fibrosis, and confirm the mechanism of Ox-LDL in rat models of obstructive jaundice. This could facilitate the present understanding of the mechanism and improve clinical treatment for liver fibrosis.

\section{Materials and methods}

Materials. In total, 72 male specific-pathogen-free Sprague-Dawley rats weighing 180-220 g were obtained from 
Table I. Liver fibrosis indicators of the rats in the control and model groups to confirm the establishment of the model.

\begin{tabular}{|c|c|c|c|}
\hline Group & Hyaluronic acid, ng/ml & Laminin, ng/ml & Procollagen III, ng/ml \\
\hline \multicolumn{4}{|c|}{ Week 1} \\
\hline A & $46.50 \pm 24.26$ & $63.05 \pm 36.25$ & $29.40 \pm 12.36$ \\
\hline $\mathrm{B}$ & $62.67 \pm 33.68$ & $52.56 \pm 25.20$ & $23.93 \pm 17.45$ \\
\hline \multicolumn{4}{|c|}{ Week 2} \\
\hline A & $38.65 \pm 13.24$ & $51.86 \pm 31.35$ & $12.56 \pm 12.20$ \\
\hline B & $58.33 \pm 19.05$ & $39.35 \pm 25.32$ & $18.20 \pm 9.46$ \\
\hline \multicolumn{4}{|c|}{ Week 3} \\
\hline A & $35.60 \pm 23.36^{\mathrm{a}}$ & $59.35 \pm 21.65^{b}$ & $25.32 \pm 13.26$ \\
\hline B & $90.64 \pm 43.16$ & $88.65 \pm 35.86$ & $53.35 \pm 26.15$ \\
\hline \multicolumn{4}{|c|}{ Week 4} \\
\hline A & $48.06 \pm 15.25^{\mathrm{a}}$ & $31.65 \pm 13.42^{b}$ & $30.63 \pm 22.47^{b}$ \\
\hline B & $365.35 \pm 132.35$ & $80.46 \pm 64.34$ & $62.91 \pm 17.56$ \\
\hline
\end{tabular}

${ }^{\mathrm{a}} \mathrm{P}<0.01$ or ${ }^{\mathrm{b}} \mathrm{P}<0.05$ vs. group B. A, control group; B, model group. Values are mean \pm standard deviation, $\mathrm{n}=6$.

A

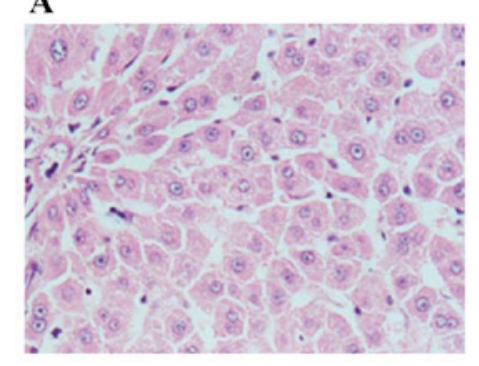

B

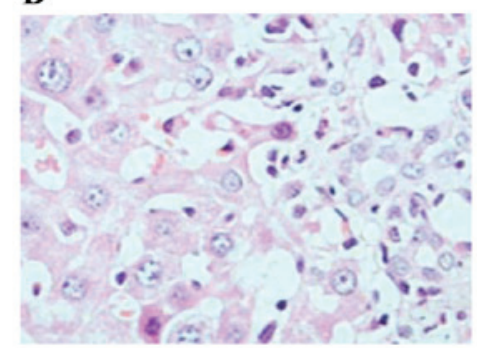

C

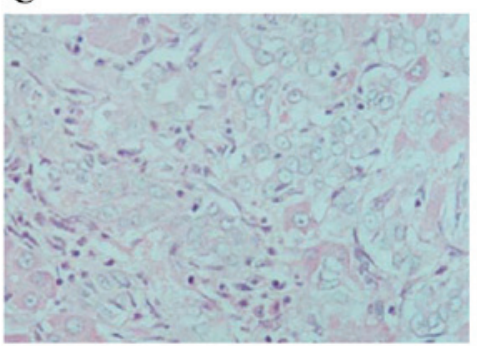

Figure 1. (A) Representative micrographs of the normal appearances of hepatocytes in group A. (B) Necrosis and the emergence of proliferation of hepatocytes in were observed group C. (C) Fibrous septamorphological abnormalities of hepatocytes in group B were evident (all magnification, x200).

A

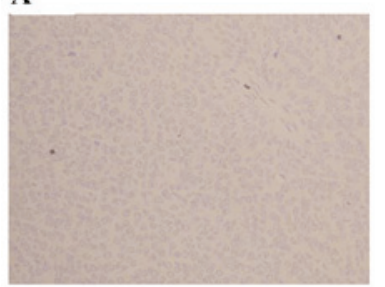

D

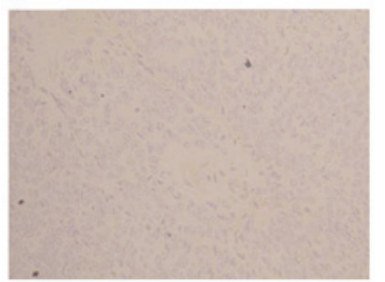

B

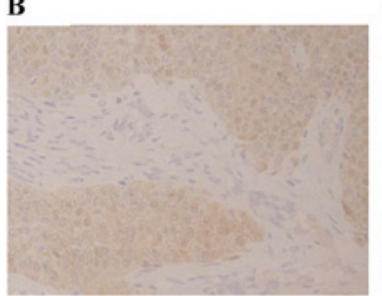

E

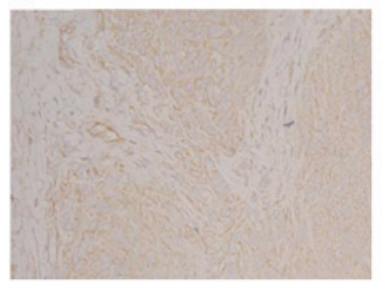

C

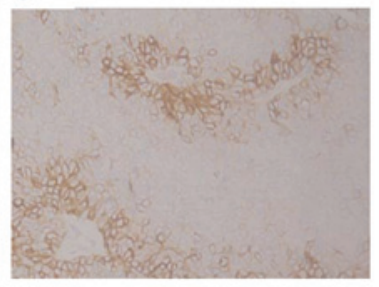

F

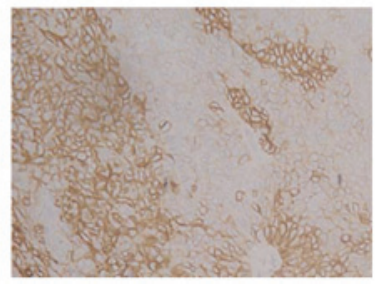

Figure 2. Expression of (A-C) SOD and (D-F) MDA in the different groups (magnification, x200). SOD staining was stronger in (C) group A compared to groups (A) B and (B) C. Group B had weaker expression of SOD compared to group C. MDA staining was stronger in (F) group B, whereas this was weaker in (D) group A. The staining intensity was graded as (A and D) 0, (B and E) 1, and (C and F) 2. SOD, superoxide dismutase; MDA, malonaldehyde.

the Experimental Animal Center of Sun Yat Sen University (Guangzhou, Guangdong, China). Six rats were housed in each cage under standard laboratory conditions. Subsequently, the rats were randomized into 3 groups: Control (group A,
24 rats), model (group B, 24 rats) and rosuvastatin groups (group C, 24 rats). Rats were fed ad libitum with a standard diet in group A, whereas rats underwent surgery with obstructive jaundice models in group B and rats were gavaged by 
Table II. SOD and MDA levels of rats from groups A and B.

\begin{tabular}{lcc}
\hline Group & SOD & MDA \\
\hline Week 1 & & \\
A & $5.28 \pm 1.56$ & $0.82 \pm 0.38$ \\
B & $4.36 \pm 2.69$ & $0.94 \pm 0.65$ \\
C & $5.22 \pm 2.36$ & $0.86 \pm 0.46$ \\
Week 2 & & \\
A & $4.86 \pm 2.58$ & $0.78 \pm 0.36$ \\
B & $4.65 \pm 1.96$ & $1.54 \pm 0.63$ \\
C & $4.95 \pm 1.38$ & $1.65 \pm 0.35$ \\
Week 3 & & \\
A & $5.66 \pm 2.65^{\mathrm{a}}$ & $0.80 \pm 0.32^{\mathrm{a}}$ \\
B & $3.86 \pm 1.56$ & $2.98 \pm 0.66$ \\
C & $4.62 \pm 1.88^{\mathrm{b}}$ & $2.18 \pm 0.62^{\mathrm{b}}$ \\
Week 4 & & \\
A & $5.45 \pm 2.38^{\mathrm{a}}$ & $0.98 \pm 0.46^{\mathrm{a}}$ \\
B & $3.26 \pm 1.68$ & $4.66 \pm 2.16$ \\
C & $3.94 \pm 2.66^{\mathrm{b}}$ & $3.82 \pm 1.36^{\mathrm{b}}$ \\
\hline
\end{tabular}

${ }^{\mathrm{a}} \mathrm{P}<0.01$ or ${ }^{\mathrm{b}} \mathrm{P}<0.05$ vs. group B. SOD, superoxide dismutase; MDA, malonaldehyde; A, control group; B, model group; C, rosuvastatin group. Values are mean \pm standard deviation, $\mathrm{n}=6$.

rosuvastatin in group $\mathrm{C}$. At weeks 1,2,3 and 4 after the model induction, 6 rats were chosen randomly from every group and anaesthetized prior to being sacrificed. To determine whether the obstructive jaundice models were successful, 1-cm length liver tissue samples were obtained through excising and subjected to hematoxylin and eosin (H\&E) staining. Blood samples were obtained from each rat for subsequent analysis of three liver fibrosis indicators. Following that, drug intervention was initiated following model induction. In addition, rats received treatment of rosuvastatin by intragastric administration in group C (batch no. 125449; AstraZeneca, London, UK) $\left(5 \mathrm{mg} \cdot(\mathrm{kg} \text { body mass })^{-1}\right.$ day $\left.^{-1}\right)$.

Analysis of serum levels by radioimmunoassay. Hyaluronic acid (HA), laminin (LN) and procollagen III (PCIII) (Guangzhou Yingwei Chuangjin Biological Engineering Co., Guangzhou, China) were examined by an automatic radioimmunoassay analyzer according to the manufacturer's protocol.

Analysis of liver fibrosis lesion by optical microscope. Paraffin-embedded samples were deparaffinized and hydrated by routine techniques. Thereafter, tissue sections were stained by $H \& E$ and observed by a light microscope.

Immunohistochemical analysis of SOD and MDA. The expression levels of SOD and MDA (Shanghai Institute of Biological Reagents Sales Co., Shanghai, China) in liver tissue were detected by immunohistochemistry. Samples were incubated with polyclonal anti-SOD and anti-MDA antibodies at a dilution of 1:50. Phosphate-buffered solution was used as the negative control. Brown staining was considered positive staining of SOD and MDA. Images were analyzed by Image-Pro plus 6.0 image analysis software (Media Cybernetics, Rockville, MD, USA). The intensity of the staining was graded as 0 (no color), 1 (yellow) and 2 (brown). In addition, the percentage of positive cells was graded as $0(<5 \%), 1(5-25 \%), 2(25-50 \%), 3(51-75 \%)$ and $4(>75 \%)$. The sum of the 2 grades served as the score for each sample.

Immunofluorescence analysis of $O x-L D L$. The expression levels of Ox-LDL (Guangzhou Yingwei Chuangjin Biological Engineering Co., Guangzhou, China) in liver tissue were detected by immunofluorescence. Samples were incubated with monoclonal anti-Ox-LDL antibody at a dilution of 1:100 according to the manufacturer's protocol. The expression levels of Ox-LDL were observed by fluorescence microscopy according to the positive ratio and intensity score of fluorescence, which had a total score of 10 points.

Statistical analysis. All data were statistically analyzed using SPSS 17.0 software (SPSS, Inc., Chicago, IL, USA). Different groups of data were analyzed with analysis of variance to detect significant differences. The significance level was set as $\alpha=0.05$. $P \leq 0.05$ was considered to indicate a statistically significant difference.

\section{Results}

Establishment of animal model. Rats in group B exhibited a poor appetite, cloudy yellow urine and less activity compared to rats in group A. To evaluate whether liver fibrosis was successful, HA, LN and PCIII were tested in the blood samples of three groups every week. As shown in Table I, group A had lower levels of HA, LN and PCIII compared to group B at weeks 3 and 4 $(\mathrm{P}<0.01)$. In addition, the liver tissues were observed by a light microscope. As shown in Fig. 1, hepatocytes were morphologically normal and orderly in group A when liver tissues exhibited degeneration, edema, necrosis, proliferation and fibrous septa morphological abnormalities at week 4 in group B. These results demonstrated that the rat model was established.

Results of indicators combining with MDA and SOD. MDA and SOD were detected by immunohistochemical analysis (Fig. 2). Table II showed the levels of MDA and SOD in group A compared with groups B and C. MDA levels were elevated $(\mathrm{P}<0.01)$ and SOD were declined $(\mathrm{P}<0.01)$ compared to group $\mathrm{A}$ at weeks 3 and 4 . Furthermore, there were reduced levels of MDA in group $\mathrm{C}$ compared with that in group $\mathrm{B}$ $(\mathrm{P}<0.05)$, but elevated levels of SOD $(\mathrm{P}<0.05)$. These findings revealed that rosuvastatin enhanced decrements in MDA and increments in SOD.

Expression of $O x-L D L$. The expression level of Ox-LDL was observed by immunofluorescence. As shown in Fig. 3, Ox-LDL was located in the hepatocyte membrane. Table III showed that the expression levels of $\mathrm{Ox}-\mathrm{LDL}$ were considerably elevated in groups $\mathrm{B}(\mathrm{P}<0.01)$ and $\mathrm{C}(\mathrm{P}<0.01)$ compared with group $\mathrm{A}$ at weeks 3 and 4 . However, the expression levels of Ox-LDL were decreased in group $\mathrm{C}$ compared with group $\mathrm{B}(\mathrm{P}<0.05)$.

Analysis of liver fibrosis. HA, LN and PCIII were tested each week in the blood samples of the rats in groups B and C. As 
Table III. Oxidized low-density lipoprotein expression level of the rats from the different groups.

\begin{tabular}{lllll}
\hline Group & Week 1 & Week 2 & Week 3 & Week 4 \\
\hline A & $1.17 \pm 0.75$ & $1.17 \pm 0.41$ & $1.17 \pm 0.75^{\mathrm{a}}$ & $1.17 \pm 0.41^{\mathrm{a}}$ \\
B & $1.00 \pm 0.63$ & $1.33 \pm 0.52$ & $3.33 \pm 1.03$ & $4.40 \pm 0.70$ \\
C & $0.83 \pm 0.41$ & $1.17 \pm 0.75$ & $2.00 \pm 0.63^{\mathrm{b}}$ & $2.33 \pm 1.21^{\mathrm{b}}$ \\
\hline
\end{tabular}

${ }^{\mathrm{a}} \mathrm{P}<0.01$ and ${ }^{\mathrm{b}} \mathrm{P}<0.05$ vs. group B. A, control group; $\mathrm{B}$, model group; $\mathrm{C}$, rosuvastatin group. Values are mean \pm standard deviation, $\mathrm{n}=24$.

Table IV. Liver fibrosis indicators of the rats in the model and following rosuvastatin treatment.

\begin{tabular}{|c|c|c|c|}
\hline Group & Hyaluronic acid, ng/ml & Laminin, ng/ml & Procollagen III, ng/ml \\
\hline \multicolumn{4}{|l|}{ Week 1} \\
\hline $\mathrm{B}$ & $62.67 \pm 33.68$ & $52.56 \pm 25.20$ & $23.93 \pm 17.45$ \\
\hline $\mathrm{C}$ & $52.67 \pm 35.66$ & $42.36 \pm 28.15$ & $22.39 \pm 15.58$ \\
\hline \multicolumn{4}{|c|}{ Week 2} \\
\hline $\mathrm{B}$ & $58.33 \pm 19.05$ & $39.35 \pm 25.32$ & $18.20 \pm 9.46$ \\
\hline $\mathrm{C}$ & $48.36 \pm 15.28$ & $32.65 \pm 21.28$ & $16.15 \pm 8.50$ \\
\hline \multicolumn{4}{|c|}{ Week 3} \\
\hline $\mathrm{B}$ & $90.64 \pm 43.16$ & $88.65 \pm 35.86$ & $53.35 \pm 26.15$ \\
\hline $\mathrm{C}$ & $70.18 \pm 33.24^{\mathrm{a}}$ & $68.36 \pm 31.52^{\mathrm{a}}$ & $43.35 \pm 18.64^{\mathrm{a}}$ \\
\hline \multicolumn{4}{|l|}{ Week 4} \\
\hline $\mathrm{B}$ & $365.35 \pm 132.35$ & $80.46 \pm 64.34$ & $62.91 \pm 17.56$ \\
\hline $\mathrm{C}$ & $258.25 \pm 128.66^{\mathrm{b}}$ & $65.24 \pm 36.35^{\mathrm{a}}$ & $45.17 \pm 21.36^{\mathrm{a}}$ \\
\hline
\end{tabular}

${ }^{a} \mathrm{P}<0.05$ and ${ }^{\mathrm{b}} \mathrm{P}<0.01$ vs. group B. B, model group; C, rosuvastatin group. Values are mean \pm standard deviation, $\mathrm{n}=6$.
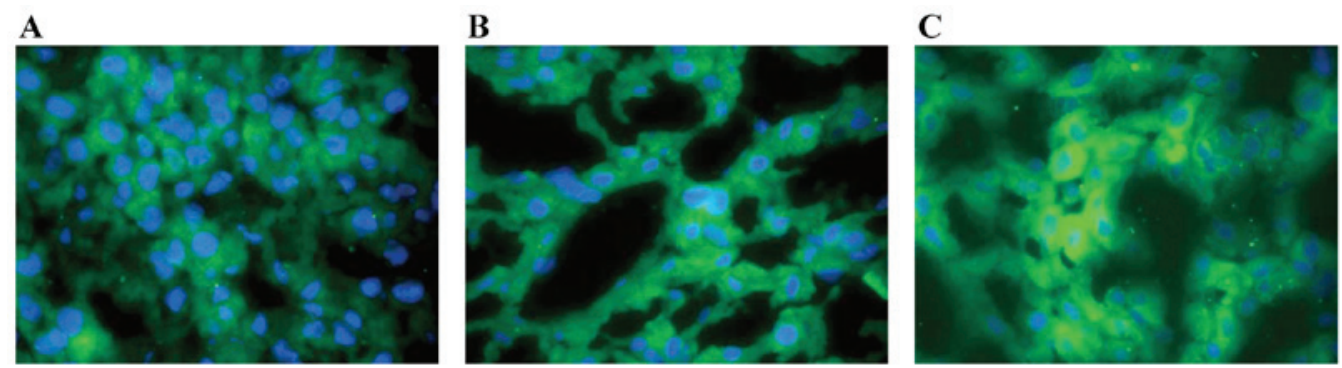

Figure 3. Expression of Ox-LDL in the different groups (magnification, $\mathrm{x} 400$ ). Ox-LDL staining was weaker in (A) group A compared to groups (B) C and (C) B. Group B had stronger expression of Ox-LDL compared to group C. The staining score of A was graded as 1, B was 3, and C was 5. Ox-LDL, oxidized low-density lipoprotein.

shown in Table IV, there were considerably lower levels of $\mathrm{HA}, \mathrm{LN}$ and $\mathrm{PCIII}$ in group $\mathrm{C}(\mathrm{P}<0.05)$ compared to group $\mathrm{B}$ at weeks 3 and 4. Fibrous septa morphological abnormalities were evident in groups $\mathrm{B}$ and $\mathrm{C}$, but these were significantly improved in group $\mathrm{C}$. These results demonstrated that treatment with rosuvastatin in rats with liver fibrosis was effective.

\section{Discussion}

Liver fibrosis is a progressive chronic disease characterized with the accumulation of ECM, which leads to serious damage to human health. However, whether the mechanism works by delaying or improving liver fibrosis remains to be elucidated.
The present study focused on the effect of rosuvastatin and its mechanism on liver fibrosis in a rat model. The model was established by bile duct ligation and gavaged administration of rosuvastatin. The study showed that the liver fibrosis indicators, HA, LN and PCIII, in group B were increased compared with that in group A. Simultaneously, the results of $\mathrm{H} \& \mathrm{E}$ staining suggested that there was more necrosis, an emergence of proliferation and the formation of fibrous septa morphological abnormalities in group B. These results demonstrated that the rat models were established.

It has been demonstrated that SOD and MDA have important roles for oxidative stress in the progression of endothelial injury and liver fibrosis. MDA is a product of 
oxidative stress, which can produce free radicals and reflect the degree of damage of liver cells. SOD can eliminate oxidative stress by removing oxygen free radicals $(10,11)$. Numerous biomolecules are closely combined with the oxidative stress on liver fibrosis. For instance, Ox-LDL appeared to stimulate liver fibrosis by causing the ECM disorder, and subsequently there is more degeneration, edema, necrosis, the emergence of proliferation and the formation of fibrous septa morphological abnormalities $(12,13)$, which are similar with the result of the present study as there was an increase of Ox-LDL in group B compared with that in group A.

Furthermore, Turer and Scherer (14) and Chen et al (15) reported that Ox-LDL has a role and is key in the regulation of the metabolism of fatty acid and glucose, which is associated with atherosclerosis. Hulthe and Fagerberg (16) reported that Ox-LDL recruited monocytes into the endothelium and caused dysfunction of artery endothelial cells via the generation of reactive oxygen species. Currently, certain abnormalities of Ox-LDL have been identified in a variety of diseases, such as liver fibrosis, however, the mechanisms remain to be elucidated. Fan et al (17) reported that Ox-LDL could induce inflammatory factors by up-regulation of autophagy via AMPK/mTOR signaling pathway. Yao et al (18) and Xiong et al (19) reported that Ox-LDL could induce cholesterol accumulation and apoptosis in macrophages by upregulating CHOP expression and the apoptosis signal-regulating kinase 1-c-Jun N-terminal kinase pathway. Samarakoon et al $(20,21)$ and Antus et al (22) reported that Ox-LDL stimulated plasminogen activator inhibitor-1 expression in human mesangial cells mediated by the transforming growth factor- $\beta$ (TGF- $\beta$ )/Smad signaling pathway. TGF- $\beta$ could activate extracellular signal-regulated kinase (ERK) in mesangial cells, and ERK was involved in the activation of $\mathrm{Smad} 2 / 3$, which increased the formation of ECM and promoted fibrosis. However, further studies are essential to elucidate the mechanism of liver fibrosis on the expression of Ox-LDL.

Changes to the MDA and SOD expression levels were also shown in the present study. Antus et al (22) and Youseff et al (23) reported that MDA is a useful marker for monitoring exacerbation-associated oxidative stress, whereas SOD is an important antioxidant enzyme for protecting cells against oxidative stress. The present study showed that the level of Ox-LDL was elevated, but the level of MDA was reduced in group B compared with group A. Furthermore, rosuvastatin inhibited the elevations of Ox-LDL and MDA but promoted the levels of SOD in group C. Therefore, the administration of the drug could markedly inhibit oxidative stress associated with liver fibrosis.

Rosuvastatin suppressed the development of liver fibrosis by inhibiting the action of HMG-CoA reductase. Resch et al (24) reported that rosuvastatin significantly reduced oxidative stress with the effect apparent after treatment for 24 weeks in vivo, which is in accordance with the result of the present study, which indicates that the administration of rosuvastatin could inhibit the decrease of fibrosis. The decrease of Ox-LDL in group $\mathrm{C}$ compared with that in group $\mathrm{B}$ indicates that rosuvastatin could exert an anti-fibrosis effect via downregulation of Ox-LDL, and subsequently prohibiting the progression of liver fibrosis.

In conclusion, the present study showed that the level of Ox-LDL could be inhibited by the administration of rosuvastatin on inhibiting oxidative stress, and rosuvastatin could improve the progression of liver fibrosis. The present study may aid in the understanding of the biological effect of rosuvastatin and improve the treatment of liver fibrosis.

\section{Acknowledgements}

The present study was supported by research grants from the Self Foundation of the Health Department of Guangxi Province of China (nos. Z2013465 and Z2013478) and the Fund of the Science and Technology Commission of Guangxi Province, China (no. 2015GXNSFAA139218).

\section{References}

1. Yue HY, Yin C, Hou JL, Zeng X, Chen YX, Zhong W, Hu PF, Deng X, Tan YX, Zhang JP, et al: Hepatocyte nuclear factor 4alpha attenuates hepatic fibrosis in rats. Gut 59: 236-246, 2010.

2. Xue $\mathrm{ZF}, \mathrm{Wu} \mathrm{XM}$ and Liu M: Hepatic regeneration and the epithelial to mesenchymal transition. World J Gastroenterol 19: 1380-1386, 2013.

3. Neuparth MJ, Proença JB, Santos-Silva A and Coimbra S: Adipokines, oxidized low-density lipoprotein, and C-reactive protein levels in lean, overweight, and obese portuguese patients with type 2 diabetes. ISRN Obes 2013: 142097, 2013.

4. Karadeniz G, Acikgoz S, Tekin IO, Tascýlar O, Gun BD and Cömert M: Oxidized low-density-lipoprotein accumulation is associated with liver fibrosis in experimental cholestasis. Clinics (Sao Paulo) 63: 531-540, 2008.

5. Li J, Fan R, Zhao S, Liu L, Guo S, Wu N, Zhang W and Chen P: Reactive oxygen species released from hypoxic hepatocytes regulates MMP-2 expression in hepatic stellate cells. Int J Mol Sci 12: 2434-2447, 2011

6. Bai YP, Hu CP, Chen MF, Xu KP, Tan GS, Shi RZ, Li YJ and Zhang GG: Inhibitory effect of reinioside $\mathrm{C}$ on monocyte-endothelial cell adhesion induced by oxidized low-density lipoprotein via inhibiting NADPH oxidase/ROS/NF-kappaB pathway. Naunyn Schmiedebergs Arch Pharmacol 380: 399-406, 2009.

7. Kose E, An T, Kikkawa A, Matsumoto Y and Hayashi H: Effects on serum uric acid by difference of the renal protective effects with atorvastatin and rosuvastatin in chronic kidney disease patients. Biol Pharm Bull 37: 226-231, 2014.

8. Li Y, Wang Q, Zhou J, Xu Q, Chu X, Sun T, Liu X and Cai S: Rosuvastatin attenuates atherosclerosis in rats via activation of scavenger receptor class B type I. Eur J Pharmacol 723: 23-28, 2014.

9. Ansari JA, Bhandari U, Haque SE and Pillai KK: Enhancement of antioxidant defense mechanism by pitavastatin and rosuvastatin on obesity-induced oxidative stress in Wistar rats. Toxicol Mech Methods 22: 67-73, 2012.

10. Aktas C, Kanter M, Erboga M, Mete R and Oran M: Melatonin attenuates oxidative stress, liver damage and hepatocyte apoptosis after bile-duct ligation in rats. Toxicol Ind Health 30: 835-844, 2014

11. Ezhilarasan D, Karthikeyan S and Vivekanandan P: Ameliorative effect of silibinin against $\mathrm{N}$-nitrosodimethylamine-induced hepatic fibrosis in rats. Environ Toxicol Pharmacol 34: 1004-1013, 2012.

12. Hong HK, Song CY, Kim BC and Lee HS: ERK contributes to the effects of Smad signaling on oxidized LDL-induced PAI-1 expression in human mesangial cells. Transl Res 148: 171-179, 2006.

13. Lee HS and Song CY: Oxidized low-density lipoprotein and oxidative stress in the development of glomerulosclerosis. Am J Nephrol 29: 62-70, 2009.

14. Turer AT and Scherer PE: Adiponectin: Mechanistic insights and clinical implications. Diabetologia 55: 2319-2326, 2012.

15. Chen Z, Li S, Zhao W, Chen X and Wang X: Protective effect of co-administration of rosuvastatin and probucol on atherosclerosis in rats. Can J Physiol Pharmacol 92: 797-803, 2014.

16. Hulthe J and Fagerberg B: Circulating oxidized LDL is associated with subclinical atherosclerosis development and inflammatory cytokines (AIR Study). Arterioscler Thromb Vasc Biol 22: 1162-1167, 2002 
17. Fan X, Wang J, Hou J, Lin C, Bensoussan A, Chang D, Liu J and Wang B: Berberine alleviates ox-LDL induced inflammatory factors by up-regulation of autophagy via AMPK/mTOR signaling pathway. J Transl Med 13: 92, 2015.

18. Yao S, Zong C, Zhang Y, Sang H, Yang M, Jiao P, Fang Y, Yang N, Song G and Qin S: Activating transcription factor 6 mediates oxidized LDL-induced cholesterol accumulation and apoptosis in macrophages by up-regulating CHOP expression. J Atheroscler Thromb 20: 94-107, 2013.

19. Xiong G, Li L, Sun S, Li T, Liao D, Shu C and Tuo Q: Subcellular localization of DAXX influence ox-LDL induced apoptosis in macrophages. Mol Biol Rep 41: 7183-7190, 2014.

20. Samarakoon R, Overstreet JM and Higgins PJ: TGF- $\beta$ signaling in tissue fibrosis: Redox controls, target genes and therapeutic opportunities. Cell Signal 25: 264-268, 2013.

21. Samarakoon R, Overstreet JM, Higgins SP and Higgins PJ: TGF- $\beta 1 \rightarrow$ SMAD/p53/USF $2 \rightarrow$ PAI- 1 transcriptional ax is in ureteral obstruction-induced renal fibrosis. Cell Tissue Res 347: $117-128,2012$
22. Antus B, Harnasi G, Drozdovszky O and Barta I: Monitoring oxidative stress during chronic obstructive pulmonary disease exacerbations using malondialdehyde. Respirology 19: 74-79, 2014.

23. Youseff BH, Holbrook ED, Smolnycki KA and Rappleye CA: Extracellular superoxide dismutase protects Histoplasma yeast cells from host-derived oxidative stress. PLoS Pathog 8: e1002713, 2012.

24. Resch U, Tatzber F, Budinsky A and Sinzinger H: Reduction of oxidative stress and modulation of autoantibodies against modified low-density lipoprotein after rosuvastatin therapy. Br J Clin Pharmacol 61: 262-274, 2006. 